\title{
Changes in Blood Flow in the Skin and Muscle with Phototherapy
}

\author{
Paul Y. K. Wu, ${ }^{[24]}$ Woon H. Wong, Joan E. Hodgman, and Norman Levan \\ Departments of Pediatrics and Medicine (Dermatology), University of Southern California School of Medicine and the \\ Los Angeles County-University of Southern California Medical Center, Los Angeles, California, USA
}

\begin{abstract}
Extract
The electrocapacitance plethysmograph with local counterpressure was adapted for measurement of total blood flow and blood flow through the muscle and skin in 20 icteric preterm infants. Measurements were recorded from each infant, before and during phototherapy. In 10 infants in study $I$, the incubator was set to maintain skin temperature of $36.5^{\circ}$ initially and during phototherapy and no attempt was made to alter the incubator settings. Total blood flow was found to increase by a mean of $116 \%$ due primarily to increased skin blood flow $(224 \%)$ and to a lesser extent, muscle blood flow $(35 \%)$. Concomitant increases in heart rate, respiration rate, skin and incubator temperature were found, but no change was observed in rectal temperature. In 10 infants in study II, the skin temperature was kept constant at $36.5^{\circ}$ before and during phototherapy by adjustment of incubator temperature. Significant increases, but of lesser magnitude, were observed in skin $(52 \%)$ and total blood flow $(37 \%)$, whereas muscle blood flow remained unchanged. Rectal temperature fell by a mean of $0.6^{\circ}$ and respiration rate and heart rate remained unchanged.
\end{abstract}

\section{Speculation}

During phototherapy both an increase in surface temperature and the direct effect of light results in augmentation of total blood flow. Increasing skin temperature appears to affect blood flow in both skin and muscle, whereas the direct effect of light from phototherapy lamps appears to affect skin blood flow only and to a lesser degree. The augmentation in skin blood flow should increase the efficiency of phototherapy. However, in small or sick infants the metabolic demands due to increasing temperature may outweigh any benefit from increasing blood flow.

Although phototherapy has been used successfully to prevent and treat hyperbilirubinemia $[6-8,14,16]$, little is known of the photobiology of the skin of neonates when they are subjected to such therapy. The reactions between the exposure of the infant to the radiation and the occurrence of photo-oxidation of bilirubin have not been clearly explained, although the process of photo-oxidation is stated to occur in the skin [18]. The rate of photo-oxidation would depend not only on the duration, irradiance, and wavelength of the radiation, but also on the concentration of bilirubin in the skin. The rate of clearance of bilirubin degradation products from the skin should be related to blood flow.

One of the effects on the skin of exposure to nonionizing electromagnetic radiations, in both the far and near UV range, is vasodilation $[12,13]$. This may be due to a photochemical reaction which leads to the 
Table I. Incubator irradiance through single layer plexiglass cover

\begin{tabular}{cc}
\hline Wave band, $\mathrm{nm}$ & Irradiance, $\mathrm{mW} / \mathrm{cm}^{2}$ \\
\hline$<360$ & 0.001 \\
$360-420$ & 0.115 \\
$420-460$ & 0.245 \\
$460-500$ & 0.227 \\
$500-650$ & 0.484 \\
$>650$ & 0.348 \\
& 1.42 \\
\hline
\end{tabular}
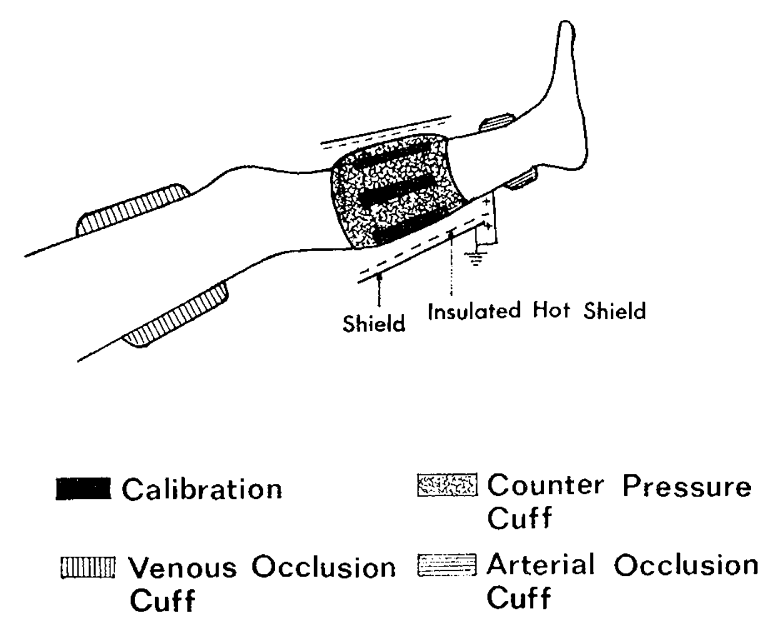

Fig. 1. Diagram of arrangement of arterial and venous pneumatic occlusion cuffs for measurement of total blood flow. Counterpressure to occlude skin blood flow was applied in the pneumatic cuff directly beneath the capacitance plethysmograph.

liberation of a mediator substance, " $\mathrm{H}$ " substance, or histamine-like dilator substance, which diffuses to the blood vessels in the dermis and causes vasodilation [12, 13]. Whether phototherapy, which involves exposure to energies in the visible light range as well as the near UV range of the electromagnetic spectrum, can cause similar changes in the infant is not known. In order to study the effects of phototherapy on skin blood flow in newborn infants, the electrocapacitance plethysmograph with local counterpressure [9] was adapted for measurement of total blood flow and skin blood flow in the calf of newborn preterm infants.

\section{Materials and Methods}

Twenty icteric preterm infants, mean birth weight $1,691 \pm 264.7 \mathrm{~g}$, gestational age $35 \pm 1.4$ weeks, and postnatal age $5 \pm 1.5$ days who were not otherwise ill, and were scheduled for treatment with phototherapy, were selected for blood flow measurements. Two sets of measurements were recorded from each infant: once before and once after at least $1 \mathrm{hr}$ of exposure to phototherapy. The light source was from 10 GE 20-W daylight fluorescent lamps mounted over the incubator to provide an illumination intensity of 450 foot-candles at the infant level. The spectrum of wavelengths emitted by these lamps ranged from $300-700 \mathrm{~nm}$ with principal peaks of emission at 436, 546, and $578 \mathrm{~nm}$. Measurement of irradiance was made with a Kendall Mark IV radiometer and the results are tabulated in Table I. All infants were kept naked in incubators except that the infants' eyes were covered with eye pads. The initial recordings were taken $0.5 \mathrm{hr}$ postprandially, when the infants were asleep or quiet. One hour on phototherapy was allowed to elapse before the second set of blood flow measurements were taken. Temperatures were recorded by telethermisters attached to a multichannel Yellow Spring recorder.

Total blood flow and blood flow through the skin and muscle were recorded by electrocapacitance plethysmograph with local counterpressure as described by Hyman et al. $[9,10]$. Modifications were made in the size of the pressure cuffs and the arterial, venous, and counterpressures in the system in order to adapt it for use in the premature infant, as described below (Fig. l). The foot was allowed to rest on a pad at or slightly above the level of the heart and an arterial occlusive cuff $(2 \mathrm{~cm})$ was placed around the ankle. Inflation of this cuff to $90 \mathrm{~mm} \mathrm{Hg}$ at the onset of the recording period excluded circulation to the foot. A venous occlusion cuff, $3.5 \mathrm{~cm}$ wide, was placed around the lower third of the thigh and inflated rapidly from a reservoir to the level at which optimal flows were recorded (usually $30-60 \mathrm{~mm} \mathrm{Hg}$ ). A special pneumatic cuff, for counterpressure and calibration, $3-3.5 \mathrm{~cm}$ wide, was wrapped around the calf directly beneath the "hot screen" of the capacitance. This arrangement made it possible to measure blood flow with the capacitance plethysmograph during application of graded counterpressure in the pneumatic cuff. Plethysmograph without counterpressure measures blood flow through all the tissues of the calf, whereas, when the critical counterpressure, which was $20 \mathrm{~mm} \mathrm{Hg}$ in these premature infants, is applied, only the blood flow through deeper structures is measured.

\section{Results}

Three sets of studies were performed. In the first set of studies involving 10 infants, control values for total blood flow and blood flow in the skin and muscles were 
initially recorded with the infant at rest with skin temperature at $36-36.5^{\circ}$ and rectal temperature at $36.5-$ $37.5^{\circ}$. Incubator temperature, heart rate, and respiration rate were also recorded. The infants were then put under the phototherapy lamps for $\mathrm{l}$ hr after which a second set of readings were taken. No attempt was made to alter incubator settings during the study.

Total blood flow was found to increase by a mean of $116 \%$ during phototherapy (Fig. 2). The mean increment in skin blood flow was $224 \%$, whereas that of the muscle was only $35 \%$. Concomitant increases in heart rate, respiration rate, skin temperature, and incubator temperature were observed. No significant change was observed in rectal temperature (Table II).

In the second set of studies involving another group of 10 infants, the skin temperature of the infants was

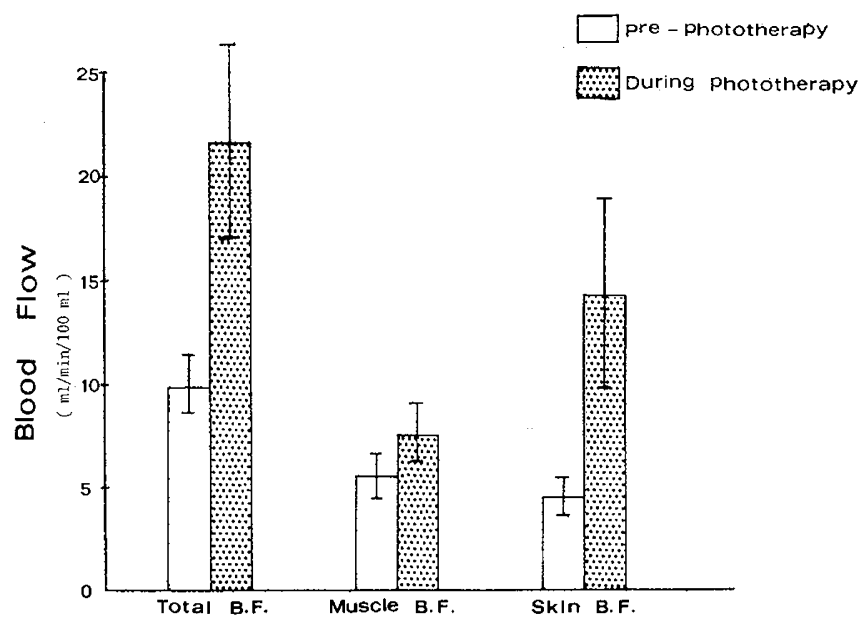

Fig. 2. Change in measurements of total, muscle, and skin blood flow before and during phototherapy in 10 infants (study $I$ ). In this study no alterations were made in incubator temperature to counteract observed concomitant rise in skin temperature and incubator temperature. kept constant at $36.5^{\circ}$ both before and during phototherapy by adjustment of incubator temperature. Blood flow measurements, heart rate, respiration rate, and skin, rectal, and incubator temperatures were recorded as above. Total blood flow was observed to be increased by a mean of $37 \%$ during phototherapy. This increase was due solely to increase in skin blood flow, which increased by $52 \%$, with no significant change in muscle blood flow (Fig. 3).

In order to maintain the skin temperature at $36.5^{\circ}$ during phototherapy, the temperature in the incubator had to be lowered in every instance from the prephototherapy setting. The range of change varied from $-5.5^{\circ}$ to $-0.5^{\circ}$ with a mean of $1.4^{\circ}$. In each infant, concomitant slight fall in rectal temperature was observed, mean $\pm 1 \mathrm{sD}=0.6 \pm 0.2^{\circ}$. No significant change was observed in heart rate and respiration rate (Table III).

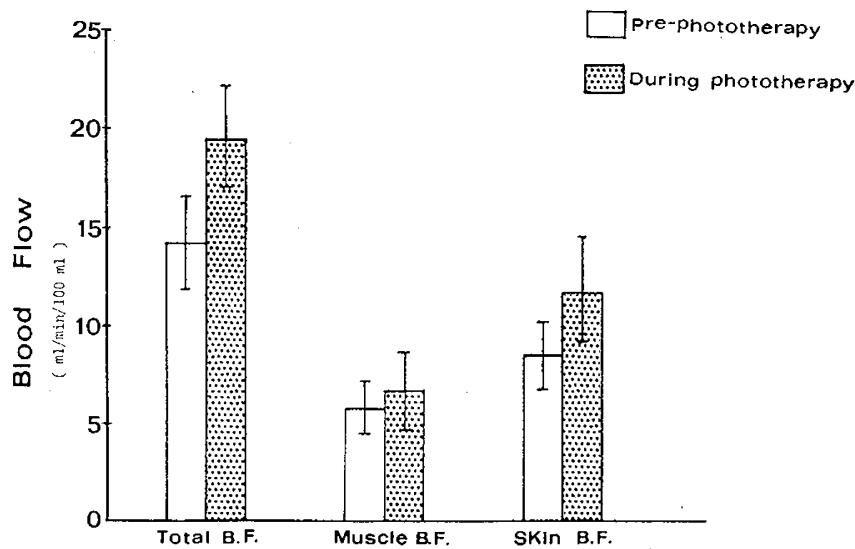

Fig. 3. Change in measurement of total, muscle, and skin blood flow before and during phototherapy in 10 infants (study In). In this study the incubator temperature was altered to maintain skin temperature at $36.5^{\circ}$ both before and during phototherapy.

Table II. Study I: changes in blood flow, skin, rectal, and incubator temperatures, respiration, and heart rate during phototherapy with no changes in initial incubator setting

\begin{tabular}{|c|c|c|c|c|}
\hline & Prephototherapy, mean $\pm 1 \mathrm{sD}$ & Phototherapy, mean $\pm 1 \mathrm{sD}$ & $\Delta$, mean $\pm \mathrm{sD}$ & $t$ Test, $P$ \\
\hline \multicolumn{5}{|c|}{ Blood flow, $\mathrm{ml} / 100 \mathrm{ml}$ tissue $/ \mathrm{min}$} \\
\hline Total & $9.98 \pm 1.40$ & $21.73 \pm 5.18$ & $+11.75 \pm 4.06$ & $<0.01$ \\
\hline Muscle & $5.58 \pm 1.22$ & $7.43 \pm 1.60$ & $+1.85 \pm 1.01$ & $<0.02$ \\
\hline Skin & $4.40 \pm 1.0$ & $14.31 \pm 4.92$ & $+9.90 \pm 4.34$ & $<0.01$ \\
\hline \multicolumn{5}{|l|}{ Temperature, ${ }^{\circ} \mathrm{C}$} \\
\hline Skin & $36.1 \pm 0.6$ & $36.7 \pm 0.8$ & $+0.7 \pm 0.3$ & $<0.05$ \\
\hline Rectal & $36.4 \pm 0.7$ & $36.7 \pm 0.8$ & $+0.3 \pm 0.3$ & N.S. ${ }^{1}$ \\
\hline Incubator & $33.9 \pm 1.4$ & $35.4 \pm 1.6$ & $+1.5 \pm 0.9$ & $<0.05$ \\
\hline Respiratory rate/min & $47.0 \pm 9.0$ & $62.0 \pm 11.0$ & $+15.0 \pm 4.0$ & $<0.01$ \\
\hline Heart $\mathrm{rate} / \mathrm{min}$ & $143.0 \pm 3.0$ & $162.0 \pm 10.0$ & $+18.0 \pm 9.0$ & $<0.01$ \\
\hline
\end{tabular}

1 N.S. : not significant. 
Table III. Study II: changes in blood flow, skin, rectal, and incubator temperatures, respiration, and heart rate during phototherapy with skin temperatures servocontrolled at $36.5^{\circ}$

\begin{tabular}{|c|c|c|c|c|}
\hline & Prephototherapy, mean \pm 1 sD & Phototherapy, mean $\pm 1 \mathrm{sD}$ & $\Delta$, mean $\pm 1 \mathrm{sD}$ & $t$ Test, $P^{1}$ \\
\hline \multicolumn{5}{|c|}{ Blood flow, $\mathrm{ml} / 100 \mathrm{ml}$ tissue $/ \mathrm{min}$} \\
\hline Total & $14.30 \pm 2.45$ & $19.48 \pm 2.90$ & $+5.17 \pm 1.70$ & $<0.01$ \\
\hline Muscle & $5.84 \pm 1.40$ & $6.73 \pm 2.11$ & $+0.90 \pm 0.77$ & N.S. \\
\hline Skin & $8.46 \pm 1.90$ & $12.74 \pm 2.97$ & $+4.28 \pm 2.08$ & $<0.01$ \\
\hline \multicolumn{5}{|l|}{ Temperature, ${ }^{\circ} \mathrm{C}$} \\
\hline Skin & 36.5 & 36.5 & 0 & \\
\hline Rectal & $37.0 \pm 0.4$ & $36.20 \pm 0.8$ & $-0.6 \pm 0.2$ & $<0.02$ \\
\hline Incubator & $35.6 \pm 1.8$ & $34.0 \pm 1.0$ & $-1.4 \pm 1.5$ & $<0.02$ \\
\hline Respiratory $\mathrm{rate} / \mathrm{min}$ & $48.0 \pm 7.0$ & $47.0 \pm 7.0$ & $+2.0 \pm 3.0$ & N.S. \\
\hline Heart rate $/ \mathrm{min}$ & $139.0 \pm 4.0$ & $141.0 \pm 4.0$ & $+1.0 \pm 1.5$ & N.S. \\
\hline
\end{tabular}

I N.S. : not significant.

Table $I V$. Changes in pre- and postprandial total blood flow

\begin{tabular}{|c|c|c|c|}
\hline \multirow{2}{*}{ Case } & \multirow{2}{*}{$\begin{array}{l}\text { Preprandial, } \\
\mathrm{ml} / \mathrm{min} / 100 \mathrm{ml}\end{array}$} & \multicolumn{2}{|c|}{ Postprandial, $\mathrm{ml} / \mathrm{min} / 100 \mathrm{ml}$} \\
\hline & & $0.5 \mathrm{hr}$ & $1.5 \mathrm{hr}$ \\
\hline 1 & 15.48 & 13.57 & 14.62 \\
\hline 2 & 10.15 & 9.09 & 10.42 \\
\hline 3 & 12.51 & 10.38 & 12.95 \\
\hline 4 & 9.06 & 7.62 & 12.76 \\
\hline 5 & 10.60 & 8.40 & 7.85 \\
\hline 6 & 16.86 & 15.85 & 11.81 \\
\hline 7 & 9.13 & 9.96 & 10.05 \\
\hline 8 & 10.44 & 11.60 & 11.25 \\
\hline \multirow{2}{*}{ mean $\pm \mathrm{sD}$} & 11.78 & 10.81 & 11.46 \\
\hline & \pm 2.75 & \pm 2.59 & \pm 1.95 \\
\hline
\end{tabular}

In order to observe the effect of postprandial variations of blood flow on our experimental design, a third set of blood flow measurements was performed on eight preterm icteric infants following the same time schedule, but without phototherapy. The results are shown in Table IV. Differences between pre- and postprandial values and between 0.5 - and 1.5 -hr postprandial values were not statistically significant $(P>0.5)$.

\section{Discussion}

Measurements of blood flow in neonates have demonstrated that peripheral blood flow varies with the maturity and postnatal age of the infant [11]. Peripheral blood flow was found to be higher on the 3rd day of life than on the 1 st or 5 th postnatal day. Because of these considerations, infants selected for this study were all preterm (mean birth wt $1,691 \pm 264.7 \mathrm{~g}$, gestational age $35 \pm 1.4$ weeks) and between the 4 th and 6 th days postnatally. The control measurements were all performed $30 \mathrm{~min}$ after feeding. The time was chosen because during this time the infants were generally quiet, since it is impossible to make accurate measurements unless the infant is still. Because the vasodilation effects of photoirradiation may persist for some time after exposure [1], the phototherapy part of the study was the second procedure in all instances. The question arises of whether the observed changes in blood flow were not related to postprandial variations. Blood flow measurements at comparable periods postprandially without phototherapy, in the third set of infants, did not show any significant changes, as seen in Table IV.

Previous studies have shown that exposure of the skin to photoirradiation in the far and near UV wavelengths leads to vasodilation followed by erythema $[1,2,4,5]$. With monochromatic UV it was further demonstrated that erythema efficiency is maximum at about $297 \mathrm{~nm}$ and $254 \mathrm{~nm}$ with a minimum at $280 \mathrm{~nm}$. In addition, there exists another broad band, with weaker efficiency, from 330 to $420 \mathrm{~nm}$. The erythema caused by the long UV wavelengths develops immediately, reaches a maximum in about $1 \mathrm{hr}$, and usually lasts for less than $24 \mathrm{hr}$ [1]. Using a Kendall Mark IV radiometer we measured the irradiance inside a C-86 isolette under phototherapy lamps. Our findings were similar to those reported in the Rohm and Haas bulletin [19] and by Yasunaga and Kean [21] that the plexiglass $G$, used in the manufacture of incubators, caused a sharp cut-off of transmission of wavelengths below $360 \mathrm{~nm}$. It seems unlikely, therefore, that the alterations in blood flow during phototherapy are caused by irradiation involving wavelengths below $360 \mathrm{~nm}$. However, our measurements did show that plexiglass will successfully pass greater than $80 \%$ of the energy lying between 360 and $420 \mathrm{~nm}$. The observed augmentation in blood flow during phototherapy may thus be related to the action of long UV radiations in this range directly on the skin. 
Another possible explanation of the changes in blood flow is the transfer of nonionizing radiant energies from phototherapy lamps to the skin, producing electron excitation. One mode of dissipation of the excitation energy would be its degradation into heat [15]. This, in turn, may cause vasodilation and increase the blood flow.

A number of factors may be involved in the observed temperature changes in the infant and the incubator in study $I$. Because the temperature of phototherapy lamps $\left(38.3-39.4^{\circ}\right)$ is higher than the skin temperature of the infant, heat loss by radiation from the infant and the incubator is diminished, and, at the same time, radiant heat from the lamps to the infant and incubator is increased. Unless compensatory mechanisms are put into operation, a rise in temperature will be expected in both. In addition to the direct effect of radiant heat, photochemical reactions in the skin together with increase in blood flow may further augment skin temperature changes. These changes may or may not affect core temperature, depending upon the infant's own homeostatic mechanisms. The observed increase in respiratory rate may be one method of losing heat by insensible water loss in order to maintain a stable core temperature in the presence of rising surface temperature $[5,19,20]$.

The question arises as to whether the observed increases in blood flow during phototherapy may be due to a response to rise in surface temperature or to a response to photochemical reactions in the skin or both. In studies of blood flow in adults, Barcroft and Edholm [3] found increased blood flow through the limbs in conjunction with rising skin temperature. Later studies by Hyman et al. [9] showed that reflex heating causing a rise of $0.5-1.0^{\circ}$ in body temperature resulted in a threefold increase in blood flow through the skin but only a slight increase in muscle blood flow. During phototherapy, with its accompanying rise in surface temperature, the increase in blood flow through the skin and muscle may be caused by the increase in surface temperature. However, when surface temperature was kept constant in study II, exposure to phototherapy resulted in an augmentation of blood flow through the skin but not the muscle. This finding demonstrates that irradiation from phototherapy lamps can cause increases in skin blood flow, although not to the degree caused by increase in surface temperature.

Inasmuch as the initiation of photo-oxidation of bilirubin occurs in the skin, one of the factors influencing efficiency of phototherapy should be rate of skin blood flow. Increase of skin temperature which, in turn, augments skin blood flow consequently should enhance the effectiveness of phototherapy. However, in small or sick infants the metabolic demands brought about by increasing temperature may outweigh any benefit from increasing blood flow. In these infants, maintenance of normal skin temperature during phototherapy should reduce this potential risk.

\section{References and Notes}

1. BACHEM, A.: Time factors of erythema and pigmentation by ultraviolet rays of different wave lengths. J. Invest. Dermatol., $25: 215$ (1955).

2. BACHEM, A.: Special review: Ultraviolet action spectra. Amer. J. Phys. Med., 35: 117 (1956).

3. Barcroft, H., AND Edholm, O. G.: Temperature and blood flow in the human forearm. J. Physiol., 104: 366 (1946).

4. Blum, H. F.: Physiological effects of sunlight on man. Physiol. Rev., 25: 483 (1945).

5. Blum, H. F.: Radiation Biology, Vol. II, p. 487 (A. Hollaender, New York, 1955).

6. Broughton, P. M. G., Rossrter, E. J. R., Warren, C. B. S., Goulis, G. AND LoRD, P. S.: Effect of blue light on hyperbilirubinemia. Arch. Dis. Childhood, 40: 666 (1965).

7. Cremer, R. J., Perryman, P. W., and Richards, D. H.: Influence of light on the hyperbilirubinemia of infants. Lancet, $i$ : 1094 (1958).

8. Hodgman, J. E., And Schwartz, A.: Phototherapy and hyperbilirubinemia of the premature. Amer. J. Dis. Child., 119: 473 (1970).

9. Hyman, G., Greeson, T., Glem, M., and Winsor, D.: Capacitance plethysmograph method for separating blood flow in muscle and skin in the human forearm. Amer. Heart J., 68: 508 (1964).

10. Hyman, C., AND Wong, W. H.: Capillary filtration coefficient in the extremities of man in high environmental temperatures. Clin. Res., 22: 251 (1968).

11. Kidd, L., Levison, H., Gemmel, P., Aharon, A., And Swyer, P. R.: Limb blood flow in the normal and sick newborn. Amer. J. Dis. Child., 112: 402 (1966).

12. Lewis, T.: The blood vessels in the human skin and their responses, p. 117 (Shaw, London, 1927).

13. Light, S.: Therapeutic electricity and ultraviolet radiation, p. 262 (The Williams \& Wilkins Co. Baltimore, 1959).

14. Lucey, J. F., Ferreiro, M., AND Hewirt, J.: Prevention of hyperbilirubinemia of prematurity by phototherapy. Pediatrics, 41: 1047 (1968).

15. MAtelsky, I.: The non-ionizing radiations. In: L. V. Galley, and G. D. Clayton: Industrial Hygiene Highlights, p. 140 Industrial Hygiene Foundation of America, Inc., Pittsburgh, 1968).

16. Obes-Pollerr, J.: La fototerapia en las hyperbilirrubinemias neonatales. Arch. Pediat. Uruguay, 38: 77 (1967).

17. Oh, W., Yao, A. C., Hanson, J. S., And Lrnd, J.: The effects of phototherapy on peripheral blood flow and insensible water loss in newborn infants. Clin. Res., 20(2): 283 (1972). 
18. Ostrow, J. D., and Branham, R. V.: Photodecay of bilirubin in vitro and in the jaundiced (Gunn) rat. Birth Defects, No. 2, p. 93 (Original Article Series, Vol. VI, 1970).

19. Transmittance and exposure stability of colorless plexiglass cast sheet, Bulletin Pl-53G (Rohm and Haas, Philadelphia, June 1971).

20. Wu, P. Y. K., and Hodgman, J. E.: Changes in insensible water loss in infants with and without phototherapy. Clin. Res., 20(2): (1972).

21. Yasunaga, S., AND Kean, E. H.: The effect of plexiglass incubators on phototherapy. J. Pediat. 81 : 89 (1972).
22. These studies were performed with informed consent in accordance with the provisions set forth in the Declaration of Helsinki.

23. This research was supported by grants from the Bireley Foundation and Health, Education and Welfare Grant no. 5S0I RR 05466-05.

24. Requests for reprints should be addressed to: P. Y. K. Wu, M.D., Women's Hospital, Los Angeles County-University of Southern California Medical Center, 1200 N. State St., Los Angeles, Calif. 90033 (USA).

25. Accepted for publication December 11, 1973. 